Produktion von Fernsehnachrichten 


\section{Aleksandra Gnach}

\section{Produktion von \\ Fernsehnachrichten}

\section{Unterschiede zwischen der deutsch- und französischsprachigen Schweiz}

Springer VS 
Aleksandra Gnach

Winterthur, Schweiz

ISBN 978-3-531-19710-4

ISBN 978-3-531-19711-1 (eBook)

DOI 10.1007/978-3-531-19711-1

Die Deutsche Nationalbibliothek verzeichnet diese Publikation in der Deutschen Nationalbibliografie; detaillierte bibliografische Daten sind im Internet über http://dnb.d-nb.de abrufbar.

\section{Springer VS}

() Springer Fachmedien Wiesbaden 2013

Das Werk einschließlich aller seiner Teile ist urheberrechtlich geschützt. Jede Verwertung, die nicht ausdrücklich vom Urheberrechtsgesetz zugelassen ist, bedarf der vorherigen $\mathrm{Zu}$ stimmung des Verlags. Das gilt insbesondere für Vervielfältigungen, Bearbeitungen, Übersetzungen, Mikroverfilmungen und die Einspeicherung und Verarbeitung in elektronischen Systemen.

Die Wiedergabe von Gebrauchsnamen, Handelsnamen, Warenbezeichnungen usw. in diesem Werk berechtigt auch ohne besondere Kennzeichnung nicht zu der Annahme, dass solche Namen im Sinne der Warenzeichen- und Markenschutz-Gesetzgebung als frei zu betrachten wären und daher von jedermann benutzt werden dürften.

Zielgruppenlektorat: Claudio Cola, Nicole Döbeli, Viviane Lichtenberger, Pirmin Roos, Konrad Weber

Layout: Dominique Ghilardi, Graphic Design

Gedruckt auf säurefreiem und chlorfrei gebleichtem Papier

Springer VS ist eine Marke von Springer DE. Springer DE ist Teil der Fachverlagsgruppe Springer Science+Business Media.

www.springer-vs.de 


\section{Dank}

Mein Dank gilt all den Menschen, ohne deren Beistand und Wohlwollen ich nicht da wäre, wo ich heute bin. Prof. Dr. Iwar Werlen danke ich für seine Unterstützung und die Bereitschaft, diese Dissertation ausser Haus zu betreuen. Prof. Dr. Daniel Perrin danke ich für die vielen fachlichen Diskussionen und dafür, dass er mich immer wieder für die Wissenschaft begeistern konnte. Besonderer Dank gilt meiner Familie, die immer Raum für meine wissenschaftliche Studie geschaffen hat. Meinem Freundeskreis danke ich fürs Gegenlesen, fürs Verständnis haben und für die guten Energien. 


\section{Inhaltsverzeichnis}

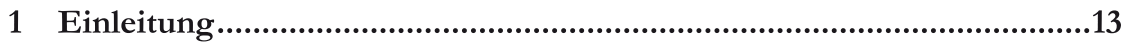

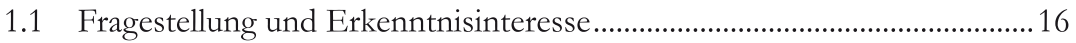

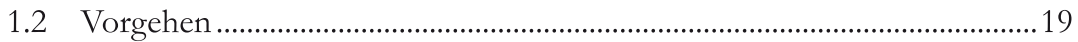

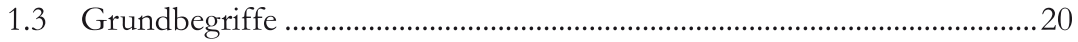

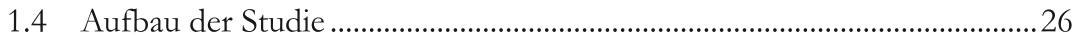

2 Kontextualisierung der untersuchten Redaktionen ............................... 27

2.1 Die SRG als Medienunternehmen mit öffentlichem Auftrag ....................27

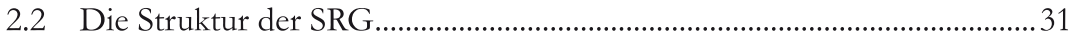

2.3 Die Unternehmenseinheiten SF und TSR ..................................................... 33

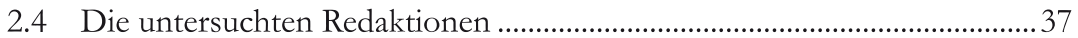

3 Relevante Disziplinen ...........................................................................41

3.1 Kommunikations- und Medienwissenschaft …………………………....... 41

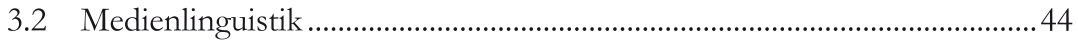

3.3 Schreibforschung ......................................................................................... 46

3.4 Fazit: Die Komplexität der journalistischen

Nachrichtenproduktion interdisziplinär erfassen........................................... 48

4 Angemessene Forschungsrahmen und Methodenansätze .......................51

4.1 Kritischer Realismus und die Domain Theory..............................................51

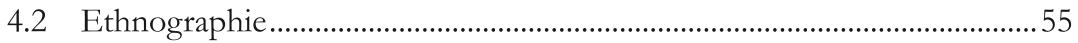

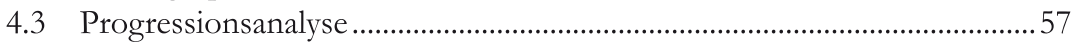

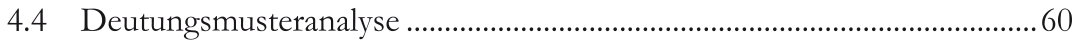

4.5 Fazit: Theoretische Begründung des Untersuchungsdesigns...................... 62

5 Forschungsstand: Sprachregionale Unterschiede in der Schweizer Medienberichterstattung........................................................................65

5.1 Komparative Studien zur Medienberichterstattung in der Schweiz...........65

5.2 Komparative Studien zu Fernsehnachrichtensendungen der SRG ............71

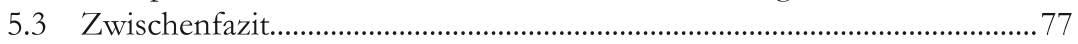

5.4 Fazit: Die Entstehung der Unterschiede prozessgerichtet erfassen...........78 


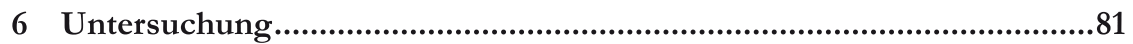

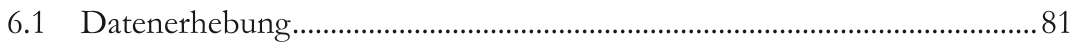

6.2 Untersuchungssample: 15 Fallstudien aus drei Redaktionen ...................... 82

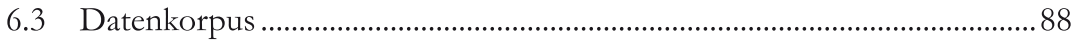

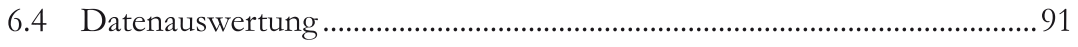

7 Ergebnisse

7.1 Ethnographie der Redaktionen................................................................. 95

7.2 Ethnographie der Nachrichtenproduktion ...............................................143

7.3 Fazit: Die Redaktionen unterscheiden sich auf den Ebenen Social Settings und Psychobiography ……………………………….......249

7.4 Deutungsmuster der Redaktionen...............................................................251

7.5 Fazit Deutungsmusteranalyse: Die Redaktionen betrachten die Welt durch unterschiedliche Brillen.......................................................................304

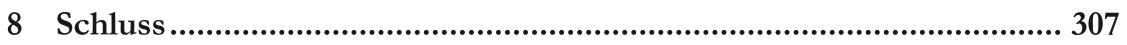

8.1 Zusammenfassung und Interpretation der Ergebnisse...............................307

8.2 Reflexion des Forschungsrahmens .............................................................. 312

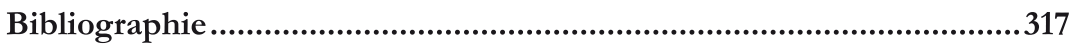




\section{Abbildungsverzeichnis}

Abbildung 1: Unternehmensstruktur SRG im Jahr 2006, Quelle:

(SRG_SSR_Idée_Suisse 2007)

Abbildung 2: Ausschnitt aus der S-Notation zum Fall OLmA; Sichtbar ist die Abfolge der [Deletionen], \{Insertionen\}, |Breaks.

Abbildung 4: Redaktionssitzung TAgESsCHAU vom 13. Oktober 2006 um 9:00 Uhr

Abbildung 5: Ausschnitt aus einer Sendungskritik der Tagesschau;

R: Redaktionsleiter, P: Produzent, X8: Redakteurin, nicht näher bestimmt (sf_ts_061101_1430_editorial_discourse)

Abbildung 6: Redaktionssitzung 10vor10 vom 23. Januar 2007 um 14:00 Uhr 119

Abbildung 7: Ausschnitt aus einer Sendungskritik von 10vor10;

R: Redaktionsleiter, P: Produzent,

X3 und X5: RedakteurInnen, nicht näher bestimmt

(sf_zvz_061218_1400_editorial_discourse)...

Abbildung 8: Redaktionssitzung JourNal vom 1. März 2007 um 9:30 Uhr .......133

Abbildung 9: Sendungskritik beim JournaL; R: Redaktionsleiter, P: Produzent, $\mathrm{X} 1$ und X2: RedakteurInnen, nicht näher bestimmt (tsr_tj_070214_0930_editorial_discourse)

Abbildung 10: Beitragstext OLMA; M: Moderation; A: Samuel Schmid, Bundesrat; O: Off-Text

Abbildung 11: Beitragstext ELEC; Quote A1: John Bolton, UNO-Botschafter USA; A2: Hugo Chavez, Präsident Venezuela; O: Off-Text..........157

Abbildung 12: Beitragstext WheA; M: Moderation; O: Off-Text 162

Abbildung 13: Beitragstext Ronc; M: Moderation, O: Off-Text, A: Bernhard Paul, Gründer Zirkus Roncalli 168

Abbildung 14: Beitragstext Rums; M: Moderation, O: Off-Text; A1:

Robert M. Gates, Designierter Verteidigungsminister USA; A2: George Bush, Präsident USA, A3: Donald Rumsfeld, Verteidigungsminister USA 


\section{Tabellenverzeichnis}

Tabelle 1: Eckdaten SF 2006. Quelle: (SRG_SSR_Idée_Suisse 2007)........................ 35

Tabelle 2: Programmleistungen SF 2006. Quelle: (SRG_SSR_Idée_Suisse 2007)..35

Tabelle 3: Eckdaten TSR 2006. Quelle: (SRG_SSR_Idée_Suisse 2007).....................36

Tabelle 4: Programmleistungen TSR 2006. Quelle:

(SRG_SSR_Idée_Suisse 2007).......................................................................... 37

Tabelle 5: Überblick über das Datenkorpus (Perrin 2011d) ........................................89

Tabelle 6: Die produzierten Beiträge (Perrin 2011d: 279) ..........................................90

Tabelle 7: Rollenverteilung und -besetzung in den untersuchten Redaktionen.......99

Tabelle 8: Sprachereignisse Redaktionssitzungen Tagesschau ..................................108

Tabelle 9: Sprachereignisse Redaktionssitzungen 10vor10 …....................................120

Tabelle 10: Sprachereignisse Redaktionssitzungen Journal..........................................137

Tabelle11: Alter, berufliche Sozialisation und Erfahrung der RedakteurInnen (Angabe in Jahren) (Perrin 2011d: 237) ..................................................246 
Abbildung 15: Beitragstext CABL; M: Moderation; O: Off-Text, A1: Jacqueline Bachmann, Stiftung für Konsumentenschutz;

A2: Rudolf Fischer, Chef Cablecom;

A3: Beatrix Zimmerli, Geschäftsführerin Inter GGA 184

Abbildung 16: Beitragstext Yout; M: Moderation; O: Off-Text, I: Interviewer;

A1: Bernhard Graser, Kantonspolizei;

A2: Michel Jungo, Förster-Lehrling;

A3: Christian Siegrist, Forstwart;

A4: Walter Mächler, Leiter Kreisschule Homberg 190

Abbildung 17: Beitragstext Temp; M: Moderation; O: Off-Text;

A1: Monika Merlo, Mutter von Unfallopfer;

A2: Gabriel Merlo, Vater von Sabina; A3: Gerhard Stoessel, Anwalt des Autofahrers

Abbildung 18: Beitragstext Wort; M: Moderation; O: Off-Text;

A: Fabio Cannavaro, Welt-Fussballer des Jahres 2006 204

Abbildung 19: Beitragstext SwIs; M: Moderation; O: Off-Text; I: Interviewer;

A1: Mario Corti, ehem. Konzernchef SAirGroup;

A2: Marcel Ospel, Konzernchef UBS

Abbildung 20: Beitragstext LeBA; M: Moderation; O: Off-Text;

A1: Demonstrant, anonym; A2: Demonstrantin, anonym

Abbildung 21: Beitragstext FAMI; M: Moderation; O: Off-Text;

A: Familienvater, anonym 224

Abbildung 22: Beitragstext GAST; M: Moderation; O: Off-Text;

A1: Antonio Miracola, propriétaire il Carosello;

A2: Claudio Casanova, vice-prés d'hôtellerie suisse;

A3: Felix Gutzwiller, conseiller national radical;

A4: Gast, anonym; A4: Gast weiblich, anonym 231

Abbildung 23: Beitragstext YogY; M1: Moderatorin; M2: Moderator;

O: Off-Text; A: Sugiarto, agent de la sécurité de l'aéroport

Abbildung 24: Beitragstext MARs; M: Moderation; O: Off-Text;

A1: Jérôme Faist, Professeur de physique expérimentale;

A2: Antoine Müller, Directeur Alpes LASER 